\title{
Characterization of Piezoresistive-Si-Nanowire-Based Pressure Sensors by Dynamic Cycling Test With Extralarge Compressive Strain
}

\author{
Liang Lou, Hongkang Yan, Woo-Tae Park, Member, IEEE, \\ Dim-Lee Kwong, Fellow, IEEE, and Chengkuo Lee, Member, IEEE
}

\begin{abstract}
A novel pressure sensor using piezoresistive silicon nanowires (SiNWs) embedded in a suspended multilayered diaphragm is investigated by a probe-based dynamic cycling test combining the standard bulge testing setup. By utilizing the high fracture stress of the $\mathrm{SiN}_{x}$ film, we explored the behavior of the SiNW under a level of extralarge compressive strain for the first time, including strain levels of more than $2.1 \%$ under the static testing and $\mathbf{1 . 5 \%}$ under the dynamic testing. Drift of the initial resistances of the SiNW was observed at different time intervals during the dynamic testing under a compressive strain of higher than $1.3 \%$, while the sensitivity of the pressure sensor basically keeps unchanged. However, there was almost no drift or degradation observed in the sensor characteristics when an equivalent point loading within the application working range is applied to the pressure sensor during the dynamic testing.
\end{abstract}

Index Terms-Fatigue, large compressive strain, piezoresistive, pressure sensor, silicon nanowire (SiNW).

\section{INTRODUCTION}

$\mathbf{T}$ HE MICROELECTROMECHANICAL systems (MEMS) pressure sensors have been used in applications ranging from the automotive industry to various biomedical devices [1], [2]. One of the earliest research efforts in biomedical applications is the development for biomedical instrumentation applications, including cardiovascular catheterization [3].

Manuscript received April 23, 2012; revised July 10, 2012; accepted August 7, 2012. Date of publication September 28, 2012; date of current version October 18, 2012. This work was supported in part by the Academic Research Committee Fund MOE2009-T2-2-011 at the National University of Singapore under Grant R-263000598112 and in part by SERC, Agency for Science, Technology and Research, under Grants 1021650084, 1021010022, and 1021520013 . The review of this paper was arranged by Editor F. Ayazi.

L. Lou is with the Department of Electrical and Computer Engineering, National University of Singapore, Singapore 117576, and also with the Institute of Microelectronics, Agency for Science, Technology and Research, Singapore 117685 (e-mail: 1lnusback1023@gmail.com).

H. Yan and C. Lee are with the Department of Electrical and Computer Engineering, National University of Singapore, Singapore 117576 (e-mail: elelc@nus.edu.sg).

W.-T. Park is with the Department of Mechanical and Automotive Engineering, Seoul National University of Science and Technology, Seoul 139-743, Korea.

D.-L. Kwong is with the Institute of Microelectronics, Agency for Science, Technology and Research, Singapore 117685.

Color versions of one or more of the figures in this paper are available online at http://ieeexplore.ieee.org.

Digital Object Identifier 10.1109/TED.2012.2214440
The ever-advancing semiconductor process technology renders making single-crystal silicon nanowires (SiNWs) via top-down fabrication, a relatively mature approach. This technology further enables the potential of shrinking down the sensor size and increasing pressure sensor sensitivity at the same time [4], [5]. While large piezoresistive effect of suspended as-grown SiNWs has been observed [6], [7], the SiNWs further contribute several merits to pressure sensors, including small footprint, low power consumption, and CMOS-compatible fabrication process [7]. To further improve the device performance, we report a new version of multilayered pressure sensor using embedded piezoresistive SiNWs. The optimization of the SiNW and the diaphragm structure are discussed with respect to the noise robustness, sensitivity, and practical applicability [8].

Considering MEMS devices with fragile and/or movable microstructures, high reliability is the essential concern to applications. So far, only limited reliability data of MEMS and SiNWs have been reported [9]-[11]. In order to explore the commercialization potential of pressure sensors using piezoresistive SiNWs, characterization of the fatigue of piezoresistive SiNWs and the other materials used in the pressure sensors is necessary. Based on atomic force microscope technique, the fatigue of SiNWs has been studied by using stress-controlled cyclic bending test. The experiments are conducted with SiNWs in the tensile region based on the freestanding suspended SiNWs; however, in the practical applications, the SiNWs usually need to be embedded and integrated with other thin films in order to realize various device functions and will experience strain in both the tensile and compressive regions. In comparison with the experiment on the suspended SiNWs, the device configuration of the multilayered pressure sensor made it more complicated and interesting to explore the reliability of the embedded SiNWs as well as the long time performance of the sensor. Moreover, in our previous study, we successfully applied an extralarge compressive strain to the SiNW by utilizing the $\mathrm{SiN}_{x}$ film with high fracture stress. Compared with the counterpart of a large-tensile-strain application by using MEMS platform, this approach makes it possible to extend the study to an unexplored compressive strain range higher than ever before [12], [13].

Here, we report the characterization of an improved multilayered pressure sensor on its sensitivity in an extralarge 


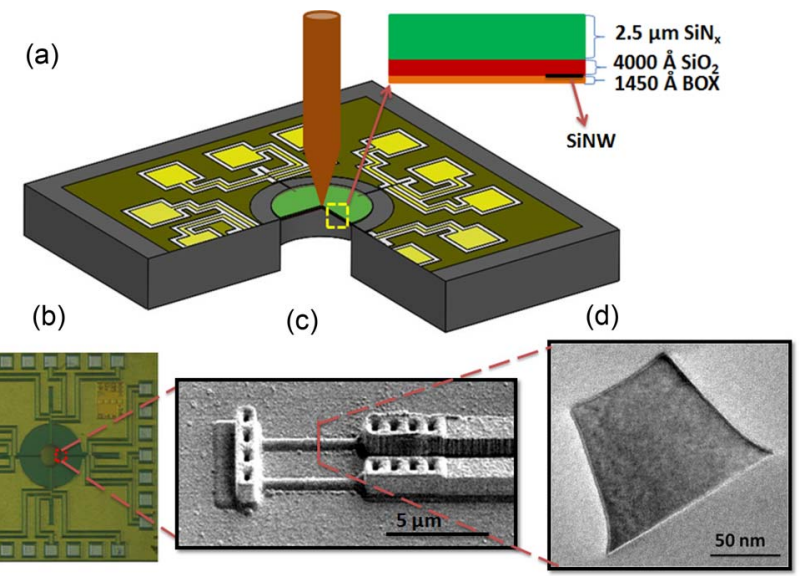

Fig. 1. (a) Schematic drawing of the pressure sensor. (b) Optical picture of the pressure sensor chip. (c) SiNW after metal formation. (d) TEM picture of the SiNW cross section.

compressive strain range and the characteristics related to fatigue concern. The displacement-based cycling test combining the standard bulge testing shows that the pressure sensor is improved with good reliability in terms of mechanical strength as well as the SiNW performance.

\section{Device Configuration and Measurement Setup}

The schematic drawing of the sensor is shown in Fig. 1(a), and the optical microscope (OM) photograph of a whole device chip is shown in Fig. 1(b). Fig. 1(c) and (d) shows the SEM picture of a 5- $\mu \mathrm{m}$ SiNW after metal deposition and its cross section. The multilayered diaphragm comprises the $\mathrm{SiN}_{x}$ layer and the $\mathrm{SiO}_{2}$ layer. The sensor chip shown in Fig. 1(b) is in square shape with dimensions of $2 \mathrm{~mm} \times 2 \mathrm{~mm}$, and it has a circular diaphragm of $200 \mu \mathrm{m}$ in diameter at the center. The yellow color refers to the $\mathrm{SiN}_{x}$ film.

The SiNWs are embedded in the diaphragm between the $4000-\AA$ oxide layer and the $1450-\AA$ BOX layer and located at the diaphragm edge to gain maximum strain when the diaphragm deforms. $\langle 110\rangle$-direction SiNWs at a dosage of $1 \times 10^{14}$ are chosen for their high sensitivity and robustness to noise [14]. The sensitivity of the sensor is defined as $S=$ $(\Delta R / R) / \Delta P$, where $S$ represents the sensitivity, $R$ is the SiNW resistance, and $\Delta P$ refers to the differential pressure uniformly applied to the diaphragm. The $2.5-\mu \mathrm{m} \mathrm{SiN}_{x}$ layer on top of the oxide layer enables the sensor with a flat diaphragm of $0.005-\mu \mathrm{m}$ central deflection and good sensitivity up to $0.32 \%$ $\left(\mathrm{lbf} / \mathrm{in}^{2}\right)^{-1}$.

The SiNWs are fabricated using the top-down approach. The photoresist patterns with respect to the nanowires have a width of $160 \mathrm{~nm}$. Then, this width is further reduced to $110 \mathrm{~nm}$ by plasma trimming, which shrinks the critical dimension to around $110 \mathrm{~nm}$. Deep reactive ion etching is conducted afterward to pattern the SiNWs. Finally, the cross section of the SiNWs is reduced to around $90 \mathrm{~nm} \times 90 \mathrm{~nm}$ by thermal oxidation, as shown in Fig. 1(d).

A tungsten needle is attached to a manipulator controlled by a position system using a piezoelectric bulk PZT actuator. The needle is deployed to push the diaphragm and transmit the
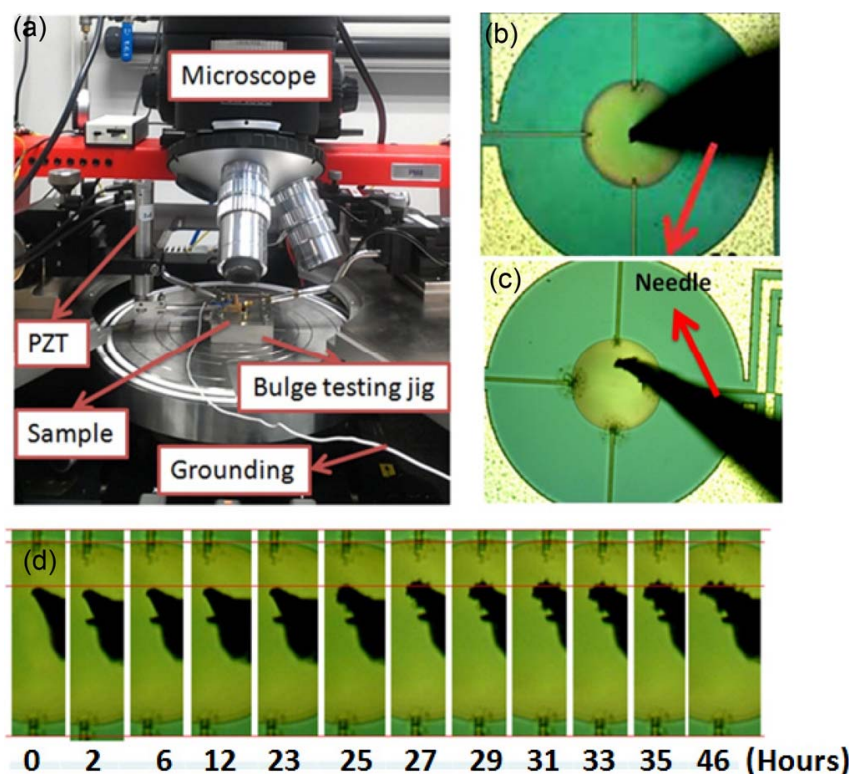

Fig. 2. (a) Testing setup. [(b) and (c)] Displacement testing with tip located (b) at the center and (c) $50 \mu \mathrm{m}$ away from the center. (d) Corresponding tip profile change against time recorded by $\mathrm{OM}$.

strain to the SiNW. Meanwhile, the electrical measurement of the SiNW resistance is conducted. The experiment is conducted at ambient temperature on a probe station platform under a microscope, as shown in Fig. 2(a). Fig. 2(b) and (c) shows the different tip positions on the diaphragm in our experiment. For static measurement, the tip moves down perpendicularly to the diaphragm at a given velocity of $1 \mu \mathrm{m} / \mathrm{s}$. For dynamic cycling measurement, the tip is set to vibrate at a frequency of $100 \mathrm{~Hz}$ along the perpendicular direction to the membrane. Finally, it is worth noting that the grounding of the needle is of importance to avoid the electrostatic force generated during the dynamic testing; otherwise, the accumulated charge due to the tip-diaphragm interaction will cause dust attachment to the tip. Fig. 2(d) shows one typical evolution of tip profiles against time without grounding during the dynamic testing. In such case, the tip-diaphragm contact position is difficult to judge.

The longitudinal strain across the diaphragm, particularly at the SiNW area, is extracted using finite-element analysis (FEA) software ABAQUS. The average strain is extracted and averaged from the corresponding elements at the SiNW area. The Young modulus and Poisson ratio values used in the modeling are obtained from the literature [15], [16], and the residual stress was extracted from warpage of the wafers, as shown in the inset table in Fig. 3. Fig. 3(a) and (b) shows the stress distribution across the diaphragm under two point loadings exactly at the center and $50 \mu \mathrm{m}$ away from the center, respectively. Fig. 3(c) shows the zoom-in area at the SiNW location at the diaphragm edge. The five-layer structure model is used to extract the maximum stress inside the $\mathrm{SiN}_{x}$ layer.

\section{RESUlts AND Discussion}

The characterization results are categorized and discussed based on the two testing methods, i.e., static testing and dynamic testing. The static testing mainly provides information on 


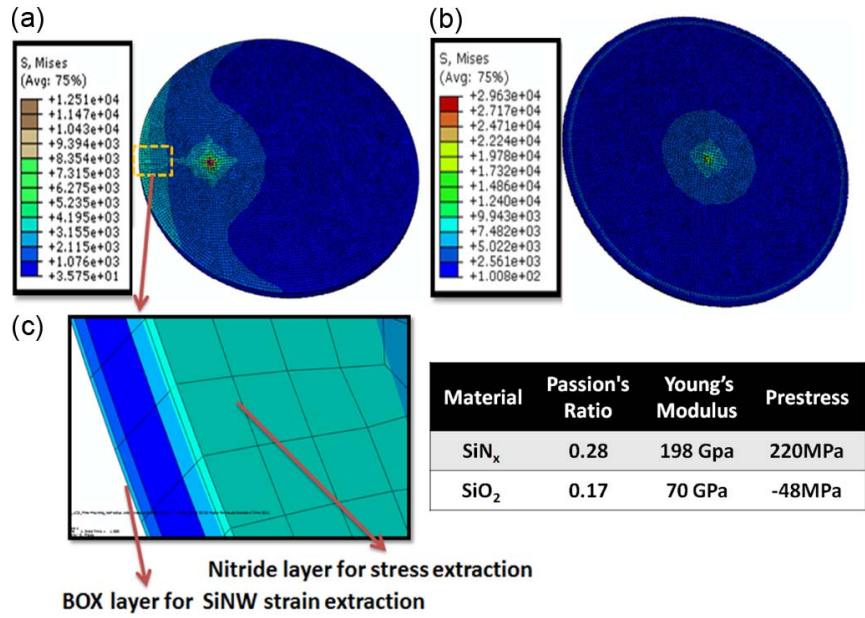

Fig. 3. FEM model of the displacement loading (a) at the center of the diaphragm and (b) near the edge of the diaphragm. (c) Zoom-in picture of the five-layered meshing of diaphragm edge. Inset table shows the parameters used in the simulation.

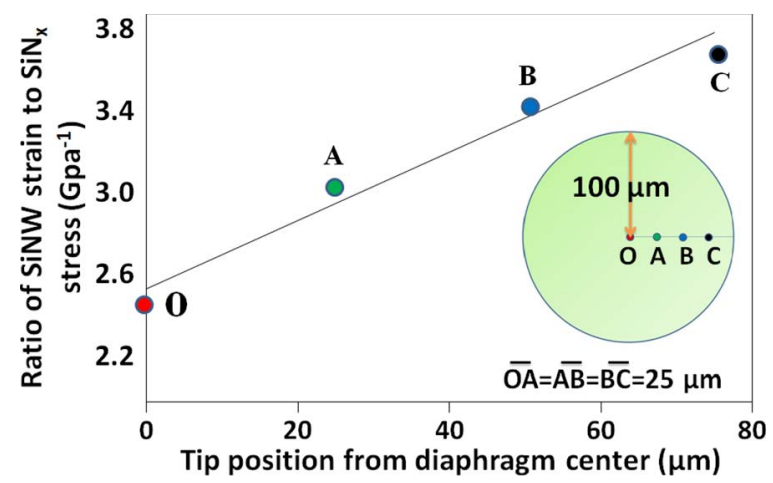

Fig. 4. SiNW resistance change against the tip displacement by static fracture testing.

the fracture behavior of the diaphragm and the SiNW resistance change under an extralarge compressive strain. These results support the dynamic testing in terms of determining the tip position and vibration amplitude.

\section{A. Static Testing}

1) Fracture Testing: The starting contact point is determined by recording the resistance as the tip moves down toward the diaphragm at a given velocity of $1 \mu \mathrm{m} / \mathrm{s}$ until the diaphragm is broken. The inset of Fig. 4 shows a typical measurement curve under this approach. The starting point and breaking point are recognized readily and immediately according to the resistance change points. More specifically, the starting point is judged when the initial resistance drops, while the breaking point is known when the resistance suddenly increases. A typical fracture measurement of the diaphragms with the tip at the center (red curve) or $50 \mu \mathrm{m}$ (blue curve) away is shown in Fig. 4. As can be seen, the diaphragm breaks when the contact points move downward to a distance of $11 \mu \mathrm{m}$ with $22 \%$ resistance change for the blue curve, in comparison to $20 \mu \mathrm{m}$ with $17 \%$ resistance change for the red one. When the tip is closer to the diaphragm edge, it is readily understood from the geometrical point of view that the diaphragm tends

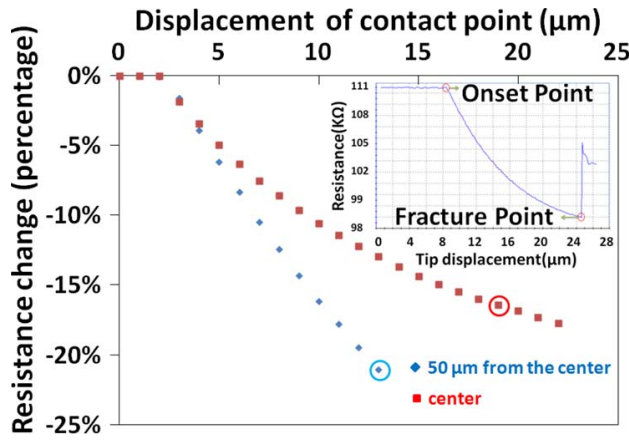

Fig. 5. Ratio of SiNW strain against maximum $\mathrm{SiN}_{x}$ stress on tip location.

to break quickly under a shorter pushing distance. In the meantime, however, it is worth noting that the SiNW exhibits a higher resistance change as well. Based on the simulation, the two circled points have identical maximum stresses in the $\mathrm{SiN}_{x}$ layer, while different resistance changes are found in the SiNWs from the experiment. This fact is explored by using FEA modeling software ABAQUS and will be discussed in more details in the next section.

The fracture stress of the composite diaphragm is decided by the toughest material, i.e., the $\mathrm{SiN}_{x}$ layer [16]. In the static testing, the fracture stress of the $\mathrm{SiN}_{x}$ is extracted as well. The $\mathrm{SiN}_{x}$ layer is found to have a fracture stress of around 4.4 GPa. Based on the maximum von Mises stress from the modeling and the theories of burst pressure [8], [17], the burst pressure is derived as around $470 \mathrm{lbf} / \mathrm{in}^{2}$ in average, indicating the strong mechanical stiffness of the diaphragm.

2) Loading Position Effect on the SiNW Strain: To more obviously reveal the dependence of the SiNW strain and the maximum stress in the $\operatorname{SiN}_{x}$ film upon the tip position, we can define a mathematic ratio $A$ as $A=\varepsilon_{\mathrm{nw}} / \sigma_{\mathrm{SiN}}$, where $\varepsilon_{\text {nw }}$ refers to the strain of the SiNW and $\sigma_{\mathrm{SiN}}$ refers to the maximum stress in the $\mathrm{SiN}_{x}$ layer. This ratio can be intuitively understood as how much compressive strain the SiNW owns when the $\operatorname{SiN}_{x}$ layer has a maximum stress of $1 \mathrm{GPa}$. It is found that this ratio depends on the tip position on top of the diaphragm. Fig. 5 shows the relationship between $A$ and the tip positions deviated from the diaphragm center toward the SiNWs, as shown in the inset of Fig. 5. We can apply these data to interpret the experimental results in Fig. 4. The resistance changes for the red circle and blue circle are $22 \%$ and $16 \%$, respectively, indicating that a larger strain is applied into the SiNW for the blue circle point than for the red one. The strains in the two SiNWs of these two points are around $2.1 \%$ and $1.5 \%$ from the simulation, which agrees with the experiments well. Thus, we can manipulate the tip position on the diaphragm to generate different maximum longitudinal strains to the SiNW before fracture. The SiNWs are able to be measured under an even larger compressive range than that reported before by us. This result is meaningful by providing a platform to investigate the behavior of SiNWs or other integratable nanowires as the sensing elements.

3) Sensitivity Versus SiNW Lengths Under Displacement Testing: The sensors with different SiNW lengths are studied using the displacement testing with the tip located $50 \mu \mathrm{m}$ away from the diaphragm center. The response curves are recorded, 


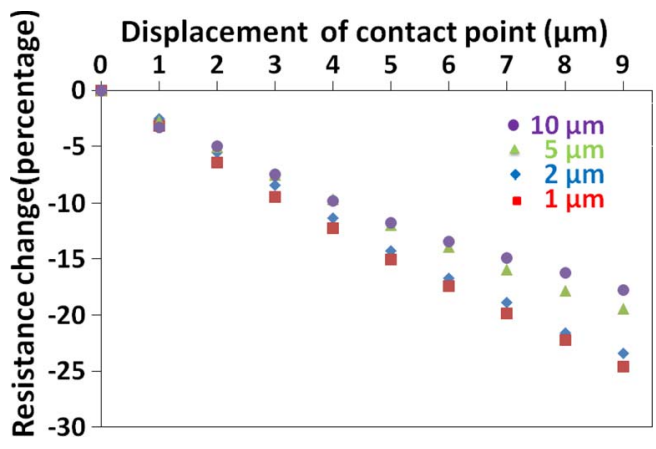

Fig. 6. Displacement testing of diaphragms with SiNWs of 1, 2, 5, and $10 \mu \mathrm{m}$ in length. (a)

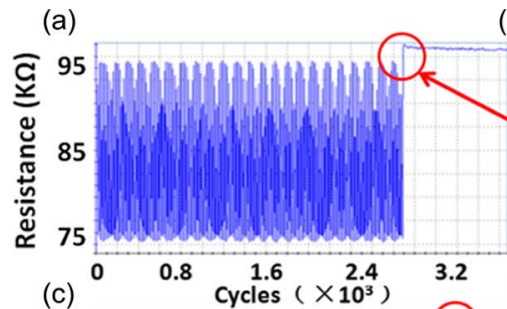

(b)

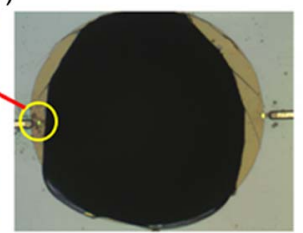

(d)

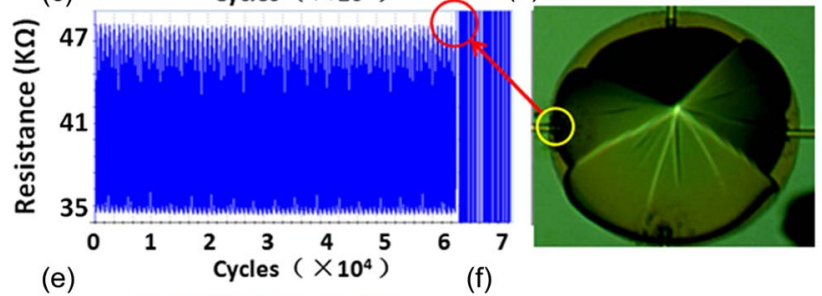

(e)

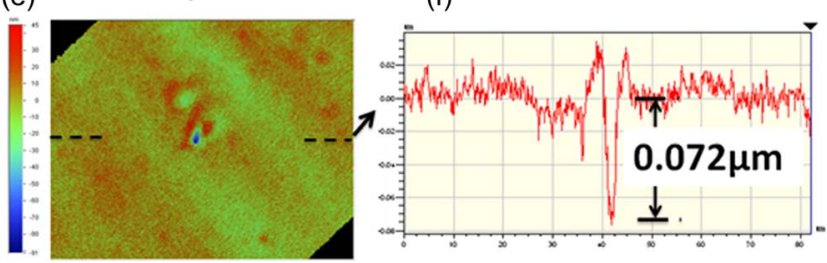

Fig. 7. [(a) and (c)] SiNW resistance change against applied cycles when the displacement is close to fracture displacement. [(b) and (d)] Optical pictures of the corresponding fractured diaphragm. (e) Zoom-in profiler of the tip-diaphragm interaction area on the diaphragm. (f) Profile across the area.

as shown in Fig. 6. As can be seen, the shorter the SiNW, the higher the measured resistance change of the sensors. This length dependence is reasonably attributed to the nonuniformly distributed strain along the SiNW. More specifically, the longer the SiNW, the lower the average strain that will be applied to the SiNW under the same displacement loading. The length effect of the SiNW is of interest in terms of fatigue in our later discussion under dynamic testing. It is possible that the longer SiNW would involve more defects than the shorter ones, which then tend to fail more easily accordingly. These static characteristics of the SiNWs with different lengths also provide a basic understanding and reference for the SiNWs and serve as the foundation for the next step study.

\section{B. Dynamic Testing}

1) Fracture Pattern: Fig. 7(a) and (c) shows the SiNW resistances against time by the dynamic cycling testing when

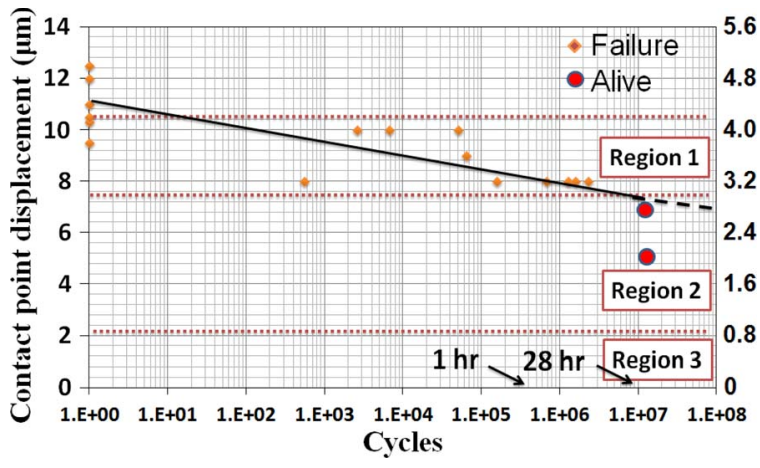

Fig. 8. $S-N$ curve of the pressure sensor under dynamic testing.

the tip is positioned $50 \mu \mathrm{m}$ away from the measured SiNW with vertical movement ranges of 10 and $9 \mu \mathrm{m}$, respectively. The stable periodical shape from each cycle of displacement shows that both the probe testing system and the pressure sensor are working properly during the vibration. The resistance changes of the SiNWs in Fig. 7(a) and (c) are 21\% and 25\%, respectively. The corresponding strain of the SiNW is extracted as $1.5 \%$. Furthermore, Fig. 7(a) and (c) shows that the diaphragms suddenly break after $2.7 \times 10^{3}$ and $6.5 \times 10^{4}$ cycles with an applied stress close to the fracture stress, respectively. The corresponding OM photographs of the fractured diaphragms are shown in Fig. 7(b) and (d). In both cases, the red circles in Fig. 7(a) and (c) show the breakage of the pressure sensor with a resistance jump; however, there is no transition observed right before fracture occurred, which indicates that the fatigue happens due to a sudden brittle fracture. In Fig. 7(a), a constant resistance appears after the periodical resistance change stops. Fig. 7(b) reveals that the fatigue happens at the edge of the diaphragm due to the originally existing flaws. It appeared in most cases in the tested samples of our experiment. This is because the edge experiences the largest stress across the diaphragm during the test. It is worth noting that the measured SiNW in the yellow circle is operational without damage, indicating that it happens to be out of the fracture path, possibly due to the reinforcement from the metal pad at the SiNW area. In Fig. 7(d), the radioactive shape of the fracture path shows the high stress around the tip contact area. Such observed shape is rational as the crack is caused by the needle-diaphragm interaction [18]. To further illustrate this point, a survived diaphragm after 9-h vibration under 6- $\mu \mathrm{m}$ dynamic testing is measured around the contact point. Fig. 7(e) shows the 3-D picture, from which an indent is obviously seen. The profile measurement reveals the depth of the indent as around $0.072 \mu \mathrm{m}$, as shown in Fig. 7(f). These data further prove the rigidity of the needle and the relative elasticity of the $\mathrm{SiN}_{x}$ film. The indent is formed as a consequence of prolonged interaction of the needle with the diaphragm, and it eventually evolves into the crack initiation site. Based on this observation, a needle with a relatively round tip is preferred in our experiment to reduce the possibility of breaking the diaphragm from the contact point, thus elongating and maximizing the dynamic testing time onto the embedded SiNWs. In fact, during most of the experiments that we conducted, the fatigue of the diaphragm happens at the diaphragm edge. By extracting the maximum von Mises 
stress from the edge of the diaphragm using simulation, we are able to derive the fatigue behavior of the $\mathrm{SiN}_{x}$ film by presenting the stress $(S)$ against the number of cycles to failure $(N)$, i.e., the $S-N$ curve, as shown in Fig. 8. More details are discussed in the following section.

2) $S-N$ Curve: The fatigue testing on the $\mathrm{SiN}_{x}$ film is conducted by placing the tip $50 \mu \mathrm{m}$ away from the diaphragm center. The displacement range and the maximum stress in the $\mathrm{SiN}_{x}$ film in the testing are shown on the left and right vertical axes of the $S-N$ curve, as shown in Fig. 8. The yellow and red dots represent the samples that fatigued before or survived after the threshold of $1 \times 10^{7}$ cycles during the dynamic testing, respectively. For the convenience of discussion, three regions are proposed in the $S-N$ curve graph as follows.

Region 1) The sensor is not able to sustain for quite many cycles up to $1 \times 10^{7}$. The diaphragm tends to break quickly under a relatively large stress to the diaphragm. The maximum stress in the $\mathrm{SiN}_{x}$ layer is more than $3.0 \mathrm{GPa}$ and lower than its average fracture stress of around $4.4 \mathrm{GPa}$. The applied strain in the SiNW is more than $1.4 \%$. Regarding the applied stress above Region 1, the diaphragm is damaged quickly.

Region 2) The diaphragm is able to survive exceeding $1 \times$ $10^{7}$ cycles when the applied stress is beyond the working range of the pressure sensor but lower than that in Region 1. The maximum stress in the $\mathrm{SiN}_{x}$ layer is lower than $3.0 \mathrm{GPa}$, and the applied strain to the SiNW is not more than $1.4 \%$. It is considered that no fatigue happens in this region.

Region 3) Obviously, no fatigue behavior happens in Region 3. This region represents the safe working range of the sensor, in which our pressure sensors can perform with good reliability.

Similar fatigue properties for the silicon nitride are observed in both macroscopic and microscopic scales, and their mechanisms are discussed as a result of the progressive accumulation of damage [19], [20]. As can be seen, the diaphragm tends to break quickly in the dynamic test when the maximum stress in the $\mathrm{SiN}_{x}$ layer comes close to its fracture stress; however, it is able to survive quite an amount of cycles when the maximum stress is below a certain critical stress for the $\mathrm{SiN}_{x}$ film. The aforementioned observations correspond to Regions 1 and 2, respectively. As mentioned, Region 3 is considered as the working range of the pressure sensor and is reasonably considered as safe operation conditions without fatigue due to the big gap of Region 2. Furthermore, the pressure sensor usually works in a very low frequency or quasi-static environment in real applications; thus, the crack growth rate in the $\mathrm{SiN}_{x}$ film is expected to be even lower by several orders of magnitude in comparison with that under cyclic loading in the dynamic testing [21]. Overall, these data serve as a further evidence of the properties of $\mathrm{SiN}_{x}$ as a brittle material and prove the endurability and reliability of the sensor. Thus, as long as the pressure sensor is protected from working in Region 2, it is able to function without breaking the mechanical structure. (a)

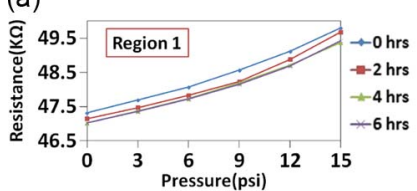

(b)

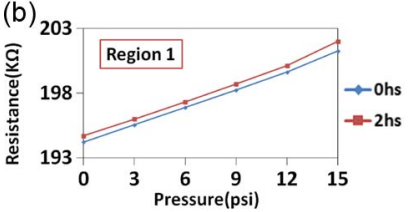

(c)

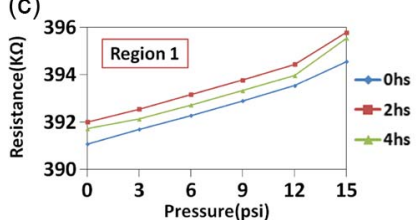

(d)

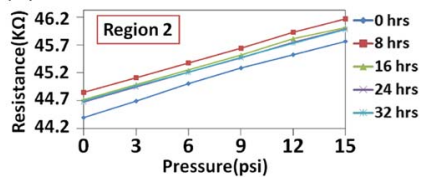

(e)

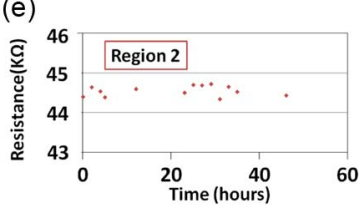

(f)

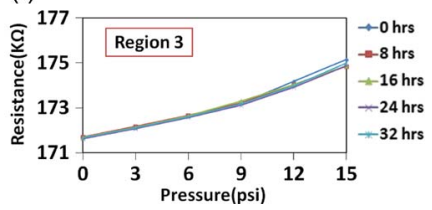

Fig. 9. (a)-(c) Bulge testing results of pressure sensors with (a) 1- $\mu \mathrm{m}$, (b) $5-\mu \mathrm{m}$, and (c) $10-\mu \mathrm{m}$ SiNWs under $8-\mu \mathrm{m}$ displacement testing. (d) Sensor result under 6- $\mu$ m dynamic testing. (e) Initial resistance against time. (f) Bulge testing results with pressure sensor under $2-\mu \mathrm{m}$ dynamic testing.

The detailed performance change during the dynamic testing is discussed in the following section.

3) Pressure Sensor Characterization During Dynamic Testing: As mentioned before, the probe-based displacement testing is used as it can exert quite high strain to the SiNW with high frequency of cycling which is difficult to be achieved in the bulge testing. However, in order to judge the fatigue of the sensor, the bulge testing is preferred because its uniform pressure application to the diaphragm is able to reasonably eliminate the uncertainty of the tip positioning onto the diaphragm in the probe-based testing. Furthermore, even if the tip can be positioned very exactly, the displacement testing may not be able to reflect the property change of the films, e.g., whether it becomes compliant or not, but this can be immediately revealed by the bulge testing because it is based on force application other than geometric deformation. Finally, the profiles of the pressure sensor during the dynamic testing are recorded to explore the profile evolution.

The experiments are conducted in the three regions that are mentioned previously using pressure sensors with SiNWs of 1, 5 , and $10 \mu \mathrm{m}$ in length. Totally, 21 samples are measured in our experiment and conducted in these three regions. Fig. 9(a)-(c) shows the typical bulge testing results in Region 1 . The dynamic testing is conducted with $8 \mu \mathrm{m}$ in amplitude, and the applied strain to the SiNW is around $1.5 \%$. A resistance drift is clearly observed at different time intervals during the testing, while no obvious dependence on the SiNW length is found for the drift. However, the sensitivities of the sensors basically keep unchanged during the dynamic testing. Fig. 9(d) shows one typical testing result in Region 2. The applied strain to SiNW is around $1.3 \%$ under the $6-\mu \mathrm{m}$ dynamic testing. The drift phenomenon is observed in this region as well. Since the sensor is considered without fatigue behavior in this region, the detailed resistance drift against time is shown in Fig. 9(e). Finally, when it falls into Region 3, the drift is interestingly found to have disappeared, as shown in Fig. 9(f). In this testing, the tip movement range 
(a)

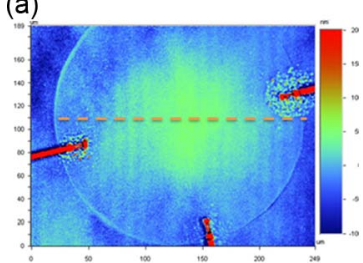

(c)

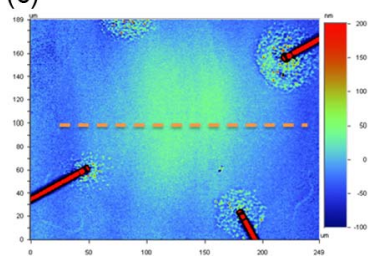

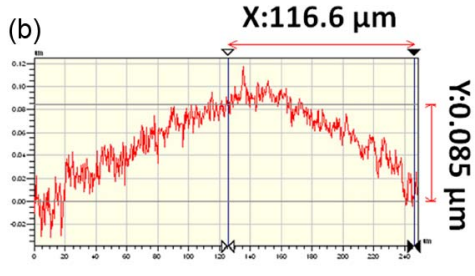

(d) $\quad X: 98.4 \mu \mathrm{m}$

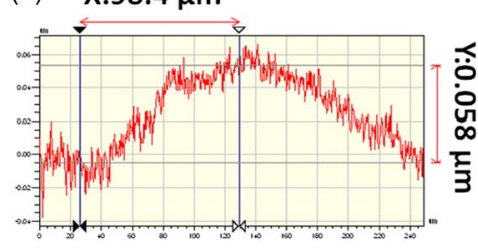

Fig. 10. (a) Two-dimensional diaphragm profile of pressure sensor before dynamic testing. (b) Recorded data of the topography across the diaphragm before the dynamic testing. (c) Two-dimensional diaphragm profile of the pressure sensor after 16-h dynamic testing. (d) Recorded data of the topography across the diaphragm after the dynamic testing.

is set as $2 \mu \mathrm{m}$, and the resistance change is around $6 \%$, which is well within the application range [15]. To explore the cause of the drift phenomenon, the diaphragm profiles at the time intervals are recorded. In our measurement, the diaphragm shows no obvious change before breaking. Fig. 10 shows a typical comparison of diaphragms before and after the dynamic testing. As can be seen from Fig. 10(a) and (c), the diaphragms present basically the same profile with good flatness. Fig. 10(b) and (d) further proves the nearly identical topography of the two diaphragms with center deflections of 0.085 and $0.058 \mu \mathrm{m}$, respectively. It is almost impossible to capture the transition state before a sound diaphragm breaks suddenly due to its brittle properties.

Combining the results of bulge testing and profile recording during the dynamic experiment, we can reasonably make some quick comments. First, the mechanical structure of the diaphragm and the SiNWs basically show no clear degradation before breaking, and there is a strong adhesion between the SiNW and its surrounding oxide; otherwise, the sensitivity of the sensor will be affected. In the report by Tang et al., the SiNWs demonstrated considerable compliant property under bending test. The SiNWs are able to be bent repeatedly in a bending strain of lower than $14 \%$ [22]. Second, the drift is of main concern, and it is related to the applied stress. As observed, the initial resistance change is usually within the range of $1 \%$. Small stress helps to eliminate the drift phenomenon.

This drift can be reasonably attributed to stress-induced charge trapping and detrapping in the silicon and oxide interface in the dynamic testing [23], [24]. The charge trapping and detrapping in the silicon-oxide interface would affect the carrier density in the SiNW channel, thus causing the drift of the initial resistance of the SiNW [24]. Due to the relatively heavy doping level inside the SiNW, slight carrier concentration change will not have significant effect on the SiNW behavior [13]. The constant sensitivity during the dynamic testing shows that the piezoresistive property in the SiNW is not affected in our pressure measurement range.
It is worth pointing out that the stress-induced trapping and detrapping phenomenon should follow a certain probability distribution regarding the stress, which means that a certain transition area must exist and should reasonably lie in the migration region from Region 2 to Region 3. It is difficult to determine the exact threshold when the stress starts to introduce trapping and detrapping into the interface, and it is supposed to be a matter of probability. However, since drift of the initial resistance can be offset by the circuitry, the results reveal that the pressure sensor is able to function continuously and properly in Region 2 and consistently within Region 3. To erase the drift effect of the sensor, deuterium incorporation is suggested to improve the interfacial oxide quality ascribed to the deuterium isotope effect. By forming the $\mathrm{Si}-\mathrm{D}$ bonds instead of $\mathrm{Si}-\mathrm{H}$ in the $\mathrm{SiO}_{2}$, such process effectively helps suppress the generation of oxide traps [25]. Furthermore, from a practical point of view, with the good waterproof property of the $\mathrm{SiN}_{x}$ film, the multilayered pressure sensor is promising as a longtime-implanted biomedical device after appropriate packaging.

\section{CONCLUSiON}

A novel pressure sensor using piezoresistive SiNWs embedded in the suspended multilayered diaphragm has been investigated by the static test and dynamic cycling test combining the probe-based testing, bulge testing, and profiler recording. In the static testing, the $\mathrm{SiN}_{x}$ layer is found to have a fracture stress of around $4.4 \mathrm{GPa}$, and the SiNW is able to be applied with a strain of more than $2.1 \%$ without breaking the diaphragm. In the dynamic testing, no obvious mechanical change of diaphragm profile is observed during the dynamic testing before breaking. A large compressive strain level up to $1.5 \%$ applied to SiNWs under dynamic testing is first reported so far. No obvious fatigue behavior is observed in the SiNWs at different compressive strain levels. The initial resistances of the SiNWs drift during the dynamic testing. The drift is found related to the applied stress, and small stress helps eliminate the drift phenomenon. However, the sensitivity of the pressure sensor maintains constant under the bulge testing approach. Overall, the characterized pressure sensor shows good reliability in terms of mechanical structure as well as the SiNW performance and is promising for biomedical applications.

\section{REFERENCES}

[1] W. J. Fleming, "Overview of automotive sensors," IEEE Sensors J., vol. 1, no. 4, pp. 296-308, Dec. 2001.

[2] A. A. Barlian, W. T. Park, J. R. Mallon, A. J. Rastegar, Jr., and B. L. Pruitt, "Review: Semiconductor piezoresistance for microsystems," Proc. IEEE, vol. 97, no. 3, pp. 513-552, Mar. 2009.

[3] Samaun, K. D. Wise, and J. B. Angell, "An IC piezoresistive pressure sensor for biomedical instrumentation," IEEE Trans. Biomed. Eng., vol. BME-20, no. 2, pp. 101-109, Mar. 1973.

[4] T. Toriyama, Y. Tanimoto, and S. Sugiyama, "Single crystal silicon nanowire piezoresistors for mechanical sensors," J. Microelectromech. Syst., vol. 11, no. 5, pp. 605-611, Oct. 2002.

[5] P. R. Nair and M. A. Alam, "Design considerations of silicon nanowire biosensors," IEEE Trans. Electron Devices, vol. 54, no. 12, pp. 34003408, Dec. 2007. 
[6] R. He and P. Yang, "Giant piezoresistance effect in silicon nanowires," Nat. Nanotechnol., vol. 1, no. 1, pp. 42-46, Oct. 2006.

[7] B. Soon, P. Neuzil, C. Wong, J. Reboud, H. Feng, and C. Lee, "Ultrasensitive nanowire pressure sensor makes its debut," in Proc. Eng., 2010, vol. 5, pp. 1127-1130.

[8] L. Lou, S. Zhang, W. T. Park, M. L. Tsai, D. L. Kwong, and C. Lee, "Optimization of NEMS pressure sensors with multilayered diaphragm using silicon nanowires as piezoresistive sensing elements," $J . M i$ cromech. Microeng., vol. 22, no. 5, pp. 550 12-550 26, May 2012.

[9] T. Tsuchiya and O. Tabata, Reliability of MEMS. Hoboken, NJ: Wiley, 2008.

[10] S. M. Ali, S. C. Mantell, and E. K. Longmire, "Experimental technique for fatigue testing of MEMS in liquids," J. Microelectromech. Syst., vol. 21, no. 3, pp. 520-522, Jun. 2012

[11] T. Namazu and Y. Isono, "Fatigue life prediction criterion for micronanoscale single-crystal silicon structures," IEEE J. Microelectromech. Syst., vol. 18, no. 1, pp. 129-137, Feb. 2009.

[12] L. Lou, W. T. Park, S. Zhang, L. Lim, D. L. Kwong, and C. Lee, "Characterization of silicon nanowire embedded in a MEMS diaphragm structure within large compressive strain range," IEEE Electron Device Lett., vol. 32, no. 12, pp. 1764-1766, Dec. 2011.

[13] J. M. Chen and N. C. MacDonald, "Measuring the nonlinearity of silicon piezoresistance by tensile loading of a submicron diameter fiber using a microinstrument," Rev. Sci. Instrum., vol. 75, no. 1, pp. 276-278, Jan. 2004.

[14] Y. Kanda, "A graphical representation of the piezoresistance coefficients in silicon," IEEE Trans. Electron Devices, vol. 29, no. 1, pp. 64-70, Jan. 1982.

[15] V. Hatty, H. Kahn, and A. H. Heuer, "Fracture toughness, fracture strength, stress corrosion cracking of silicon dioxide thin films," J. Microelectromech. Syst., vol. 17, no. 4, pp. 943-947, Aug. 2008.

[16] H. Huang, K. J. Winchester, A. Suvorova, B. R. Lawn, Y. Liu, X. Z. Hu, J. M. Dell, and L. Faraone, "Effect of deposition conditions on mechanical properties of low-temperature PECVD silicon nitride films," Mater. Sci. Eng. A, vol. 435-436, pp. 453-459, Nov. 2006.

[17] S. C. Gong and C. Lee, "Analytical solutions of sensitivity for pressure microsensors," IEEE Sensors J., vol. 1, no. 4, pp. 340-344, Dec. 2001

[18] Y. Akiniwaa, N. Miyamotob, H. Tsurua, and K. Tanakaa, "Notch effect on fatigue strength reduction of bearing steel in the very high cycle regime," Int J Fatigue, vol. 28, no. 11, pp. 1555-1565, Nov. 2006.

[19] W. H. Chuang, R. Fettig, and R. Ghodssi, "Nano-scale fatigue study of LPCVD silicon nitride thin films using a mechanical-amplifier actuator," J. Micromech. Microeng., vol. 17, no. 5, pp. 938-944, May 2007.

[20] S. Horibe, "Fatigue of silicon nitride ceramics under cyclic loading," J. Eur. Ceramic Soc., vol. 6, no. 2, pp. 89-95, Jan. 1990.

[21] J. Moffatt and L. Edwards, "Effect of frequency on ambient temperature fatigue crack growth in a silicon carbide reinforced silicon nitride composite," Int. J. Fatigue, vol. 30, no. 7, pp. 1289-1297, Sep. 2008.

[22] D. M. Tang, C. L. Ren, M. S. Wang, X. L. Wei, N. Kawamoto, C. Liu, Y. Bando, M. Mitome, N. Fukata, and D. Golberg, "Mechanical properties of Si nanowires as revealed by in situ transmission electron microscopy and molecular dynamics simulations," Nano Lett., vol. 12, no. 4, pp. 1898-1904, Apr. 2012.

[23] Y. Tu and J. Tersoff, "Structure of the silicon-oxide interface," Thin Solid Films, vol. 400, no. $1 / 2$, pp. $95-100$, Dec. 2001.

[24] V. Schmidt, S. Senz, and U. Gösele, "Influence of the $\mathrm{Si} / \mathrm{SiO} 2$ interface on the charge carrier density of Si nanowires," Appl. Phys. A, vol. 86, no. 2, pp. 187-191, Feb. 2007.

[25] A. J. Hof, E. Hoekstra, A. Y. Kovalgin, R. V. Schaijk, W. M. Baks, and J. Schmitz, "The impact of deuterated CMOS processing on gate oxide reliability," IEEE Trans. Electron Devices, vol. 52, no. 9, pp. 2111-2115, Sep. 2005.

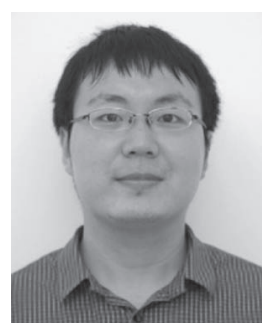

Liang Lou is currently working toward the Ph.D. degree at the National University of Singapore, Singapore.

$\mathrm{He}$ is also currently a Scientist I with the Institute of Microelectronics, Agency for Science, Technology and Research, Singapore.

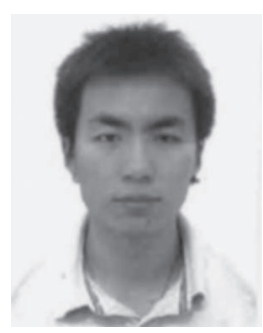

Hongkang Yan received the B.S. degree from Northeastern University, Shenyang, China, and the M.Sc. degree from the National University of Singapore, Singapore

$\mathrm{He}$ is currently with the National University of Singapore.

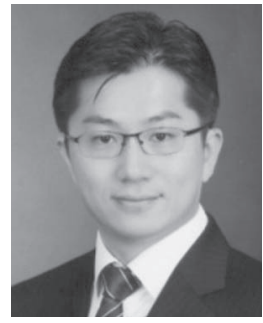

Woo-Tae Park (M'06) received the Ph.D. degree from Stanford University, Stanford, CA.

$\mathrm{He}$ is currently an Assistant Professor with the Seoul National University of Science and Technology, Seoul, Korea.

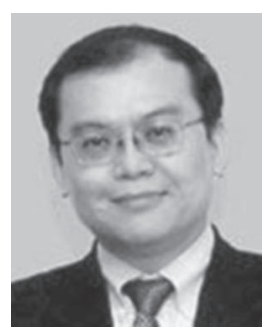

Dim-Lee Kwong (F'09) received the Ph.D. degree from Rice University, Houston, TX.

$\mathrm{He}$ is with the Institute of Microelectronics, Agency for Science, Technology and Research, Singapore, and the National University of Singapore, Singapore.

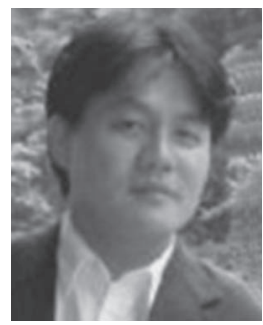

Chengkuo Lee (M'96) received the Ph.D. degree from The University of Tokyo, Bunkyo, Japan.

$\mathrm{He}$ is currently an Assistant Professor with the Department of Electrical and Computer Engineering, National University of Singapore, Singapore. 